\title{
JOGOS ELETRÔNICOS EM FOCO: ENCONTROS ENTRE OS PRINCÍPIOS DE APRENDIZAGEM E AS INTELIGÊNCIAS MÚLTIPLAS
}

\author{
Marco Antônio Bomfoco \\ Doutor em Linguística Aplicada pela PUCRS. Professor da Secretaria Municipal de \\ Educação de Porto Alegre (SMED/POA). Contato: bomfoco@ig.com.br \\ Victor de Abreu Azevedo \\ Licenciado em Educação Física, Mestre em Educação (UFSC). \\ Professor da Secretaria Municipal de Educação de Porto Alegre (SMED/POA). Membro \\ dos grupos de pesquisa Labomidia e Edumidia (UFSC). Contato: victorazev@gmail.com
}

Resumo: Neste estudo, investigamos as possibilidades de associação entre duas teorias da aprendizagem: os princípios de aprendizagem presentes nos "bons jogos” eletrônicos (PA) de James Gee e as inteligências múltiplas (IMs) de Howard Gardner. A interação foi discutida com base na noção de Domínio Semiótico (DS). A associação entre os trinta e seis PA e as oito IMs permitiu reduzir os resultados a seis grupos de itens, nos quais se destacaram, a princípio, os seguintes pontos: a) há vinte e nove PA que se relacionam com todas as IMs; e b) a inteligência interpessoal relaciona-se com trinta PA. Os resultados iniciais revelam que para que os PA possam se desenvolver a partir do jogo eletrônico dependem de sucesso num domínio completo.

Palavras-chave: jogos eletrônicos, teorias de aprendizagem, inteligências múltiplas, princípios de aprendizagem, domínio semiótico.

\section{ELECTRONIC GAMES IN FOCUS: ENCOUNTERS BETWEEN THE PRINCIPLES OF LEARNING AND THE MULTIPLE INTELLIGENCES}

\begin{abstract}
In this study, we investigated the potential association between two learning theories: learning principles present in "good games" from James Gee and multiple intelligences from Howard Gardner. This interaction was discussed based on the notion of semiotic domain. The association between thirty-six learning principles and eight multiple intelligences has reduced the results to six groups of items, in which we highlighted the following points: a) there are twenty-nine learning principles that relate to all multiple intelligences; and b) interpersonal intelligence relates to thirty learning principles. Initial results show that for the learning principles can be developed from an electronic game, the success on a complete domain is necessary.
\end{abstract}

Keywords: electronic games, learning theories, multiple intelligences, learning principles, semiotic domain.

\section{Introdução}


Pessoas em todos os estágios da vida passam horas de seu tempo de lazer jogando jogos eletrônicos (JEs) e interagindo pessoalmente no mesmo local ou de modo online com outros jogadores. Os JEs podem ser práticas longas, difíceis, desafiadoras e, apesar disso, são considerados divertidos e inspiradores. Muitos pesquisadores perguntam-se o que faz com que os JEs sejam tão interessantes. Os JEs são, pois, uma questão social e cultural significativa.

O pesquisador James Gee é pioneiro no estudo sobre a aprendizagem desenvolvida por meio dos JEs. Gee (2003) propôs que há trinta e seis princípios de aprendizagem possíveis de serem encontrados e desenvolvidos nos JEs. O autor define os JEs como domínio semiótico (DS), que, por sua vez, faz parte do DS mais amplo da vida cotidiana. Com o conceito de DS, Gee (2003:13) procura aproximar os JEs de uma definição mais ampla de letramento que abarca diferentes tipos de "letramento visual”. Assim, a partir do conceito de DS - e não mais falando em letramento na concepção tradicional de ler e escrever - considera-se que as pessoas são letradas em um domínio se forem capazes de reconhecer ou produzir significados no domínio.

De fato, no mundo contemporâneo, a linguagem articulada (falada/escrita) não é o único sistema de comunicação importante. Hoje, imagens, símbolos, gráficos, diagramas, artefatos e muitos outros símbolos visuais são significativos. Por exemplo, é importante aprender o letramento visual para "ler" as imagens em um anúncio publicitário. Ademais, palavras e imagens são integradas ou justapostas de muitas maneiras: em revistas, jornais, livros didáticos, etc.; as imagens assumem cada vez mais espaço e têm sentidos que podem ser independentes das palavras nos textos.

Para compreender as diversas formas de atuação do homem na sociedade complexa em que vivemos, tornou-se necessário o desenvolvimento de um modelo de inteligência que apresentasse uma visão pluralística da inteligência. Assim, Howard Gardner (1983) desenvolveu uma perspectiva mais ampla e pragmática da inteligência ao propor um modelo de sete inteligências básicas conhecido como teoria das inteligências múltiplas. Tanto Gee (2003) como Gardner (1983) valorizam a interação entre aprendizagem e habilidades presentes na vida cotidiana (cultura) das pessoas.

Pensando a partir da proposta de DS elaborada por Gee, vemos que na interação entre as duas teorias, o DS da vida cotidiana, o maior conjunto existente - onde ocorrem as inteligências múltiplas (IMs) -, engloba o DS dos JEs. Gardner (1983:10) afirma que um dos seus objetivos ao apresentar sua teoria foi o de examinar as implicações educacionais de uma teoria das inteligências múltiplas. Tendo em vista que Gee (2003) levantou trinta e seis princípios de aprendizagem (PA) presentes nos JEs, vinte anos após a publicação da teoria de Gardner, e considerando a importância e a popularidade dos JEs na cultura contemporânea, pareceu-nos interessante investigar como esses PA se relacionam com as IMs. Desse modo, a questão central que abordamos é: os PA presentes nos JEs e as aprendizagens que possibilitam podem auxiliar no desenvolvimento das IMs importantes para a vida cotidiana? Ou seja, o que encontramos na intersecção de ambos os domínios semióticos? Como se vê, a proposta desta pesquisa foi verificar as possibilidades de associação entre as teorias. A partir disto um primeiro esforço de análise foi realizado e é aqui apresentado.

Para responder a questão central de nossa pesquisa, desenvolvemos como metodologia da pesquisa: revisão bibliográfica, consulta a sites, observação de JEs, 
construção do modelo de interação entre ambas as propostas de aprendizagens, análise do modelo, e, por fim, as considerações sobre a pesquisa e suas referências. A pesquisa terá continuidade a partir de novas análises que devem trazer subsídios para a construção de propostas pedagógicas com os JEs. Outros pontos de vista poderão se apropriar do quadro elaborado de acordo com seus objetivos.

\section{A capacidade de aprender}

O estudo do potencial humano tem importância para a compreensão das muitas diferenças entre as pessoas e da aprendizagem em geral. Nesta seção, veremos que desde o início do século XX pesquisadores apresentam modelos da inteligência humana.

\subsection{A teoria clássica da inteligência}

De acordo com Armstrong (2009:5-6), quando Alfred Binet e Theodore Simon aceitaram, em 1904, o pedido do governo francês para elaborar uma escala métrica para avaliar a inteligência, que passou a ser conhecida como escala Binet-Simon, acabaram desenvolvendo o paradigma da inteligência que seria predominante no século XX. A escala Binet-Simon, baseada em trabalhos anteriores de Binet, foi revisada em 1908, sendo adaptada às faixas etárias dos três aos treze anos.

A finalidade do teste era identificar os estudantes problemáticos que necessitavam de educação alternativa. A partir dos esforços de ambos, surgiram, então, os primeiros testes de inteligência. Desde então, a inteligência humana foi objetivamente avaliada e reduzida a um número denominado de quociente de inteligência (QI). O aspecto mais controverso do QI é que ele representa uma inteligência que é difícil de mudar ou evoluir. Este modelo de inteligência única é conhecido hoje como a teoria clássica da inteligência. Os críticos afirmam que devido à dificuldade em se definir exatamente o que constitui a inteligência, talvez o QI deva, na realidade, exemplificar um tipo muito específico de inteligência.

A escala métrica, ainda que tenha sido um primeiro passo para a análise da inteligência, serviu para justificar o modelo do déficit principalmente por não reconhecer os múltiplos fatores responsáveis pelas diferenças individuais entre os estudantes. Possivelmente, uma visão diferenciada da situação dos estudantes franceses no início do século poderia revelar que, ao contrário de anormalidades individuais, as dificuldades de aprendizagem constatadas apontavam para a necessidade de reformas nos métodos de ensino.

\subsection{Um novo modelo para o estudo da inteligência: inteligências múltiplas}

A teoria da aprendizagem de Gardner (1983) é uma visão alternativa à teoria da inteligência tradicional. Segundo Gardner (2006), o modelo das IMs utilizou, em parte, conhecimentos que não estavam disponíveis na época de Binet: a ciência cognitiva (estudo 
da mente) e a neurociência (estudo do cérebro). Nele, a inteligência passa a ser compreendida como habilidades múltiplas.

Para Gardner (1983:8), a competência cognitiva humana é mais bem descrita em termos de um conjunto de habilidades, talentos, ou ainda competências intelectuais, que o autor denomina de inteligências, e que são relativamente autônomas. Note-se que as inteligências não operam isoladamente. Elas não existem de modo completamente independente a não ser nos casos de pessoas que sofreram danos cerebrais ou no caso dos idiotas sábios (idiots savants). Para Armstrong (2009:6), o conceito de inteligência proposto por Gardner é funcional: a inteligência é vista trabalhando na vida das pessoas nas mais variadas formas, perdendo, assim, a aura mística que ainda conservava. Em síntese, o modelo de Gardner trouxe uma visão pluralística do intelecto humano. Todavia, atente-se ao fato que a noção de “inteligências múltiplas” não é absolutamente nova. Segundo Wren e Wren (2003: 255), explicações da inteligência com base em fatores múltiplos já foram propostas por Thurstone e Guilford, que rejeitaram a noção de inteligência unitária.

O conjunto original das inteligências propostas em Gardner (1983) apresentava sete inteligências básicas. Em desenvolvimento posterior do modelo, Gardner (1993) adicionou uma oitava inteligência (naturalista) e deixou em aberto a discussão sobre a possibilidade de adotar uma nona inteligência (espiritual). Para chegar a este modelo, Gardner (1983:9) estudou amplo e não relacionado grupo de fontes: estudos de prodígios, indivíduos superdotados, pacientes com danos cerebrais, idiots savants, crianças normais, adultos normais, experts em diferentes áreas de estudo e indivíduos de diferentes culturas.

As oito inteligências são definidas como habilidades de: 1) usar a linguagem de modo competente (linguística); 2) raciocinar de modo lógico em matemática e em ciências (lógico-matemática); 3) notar detalhes do que se vê e visualizar e manipular objetos na mente (espacial); 4) compreender, criar e apreciar música e conceitos musicais (musical); 5) usar o próprio corpo habilmente (corporal-cinestésica); 6) reconhecer aspectos sutis do comportamento das outras pessoas (interpessoal); 7) ter compreensão do próprio eu (intrapessoal); e 8) reconhecer padrões e diferenças na natureza (naturalista). Essas categorias ou inteligências representam elementos que podem ser encontrados em todas as culturas, a saber: música, palavras, lógica, pinturas, interação social, expressão física, reflexão interior e apreciação da natureza. Assim, diferentemente de um estilo de aprendizagem, que é uma abordagem geral que o indivíduo pode aplicar de modo igual a qualquer conteúdo imaginável, a inteligência, para Gardner (1993), é uma capacidade com processos próprios e que está engrenada a um conteúdo específico no mundo (por exemplo, sons musicais ou padrões espaciais).

\section{Jogos eletrônicos: regras e ficção}

Com o desenvolvimento da pesquisa computacional, a partir dos anos $1950^{1}$, surgem os JEs, ocorrendo, a rigor, a interação entre regras de jogos e jogos de ficção. Desde então, como observa Juul (2005), mudou-se a forma que os jogos são jogados e percebidos pelas pessoas. Pode-se afirmar que os JEs constituem-se em uma nova forma cultural na sociedade contemporânea. 
Os JEs podem proporcionar experiências enriquecedoras, tornando-se também importante auxílio na aprendizagem tanto na escola como fora dela. De fato, há educadores que defendem a utilização dos computadores e dos JEs como ferramentas poderosas, ao propiciarem novas e ricas oportunidades de aprendizagem. Juul (2005:8) explica isto dizendo que "os jogos são, frequentemente, problemas bem estruturados, o que possibilitou seu uso em muitos campos diferentes.” Enfim, os JEs possibilitam o acesso a experiências novas e permitem que se construam modelos da realidade, ou seja, modelos simulados que tornam mais fácil desenvolver coisas no mundo real (Shaffer, 2006).

\subsection{O domínio semiótico dos jogos eletrônicos}

Para problematizar o conteúdo dos JEs, Gee (2003) desenvolve o conceito de domínio semiótico, que inclui “(...) qualquer conjunto de práticas que recrutam uma ou mais modalidades (por exemplo, linguagem oral ou escrita, imagens, equações, símbolos, sons, gestos, gráficos, artefatos, etc.) para comunicar distintos tipos de significados.” (Gee, 2003, p.18).

O autor nos traz como exemplos de DS o rap, as pinturas modernistas e os JEs do gênero tiro com visão em primeira pessoa ${ }^{2}$. De acordo com Gee, para obtermos o aprendizado de um DS qualquer, é necessário que: 1) aprendamos a experimentar o mundo de diferentes formas; 2) aprendamos a formar afiliações com os membros do DS; e 3) aprendamos a ganhar recursos necessários para os aprendizados futuros do DS e para a solução de problemas do DS, assim como de DS a ele relacionados (Gee, 2003, p 23).

Segundo Gee (2008), a afiliação com os demais membros do DS é importante já que uma "identidade social” é indispensável para a aprendizagem: "Grupos sociais existem para introduzir novatos em experiências diferenciadas e em formas de interpretação e de utilização dessas experiências para atingir metas e solucionar problemas.” (Gee, 2008, p.46). Desta forma a boa aprendizagem requer a participação em um grupo social. Este auxilia seus membros na compreensão dos propósitos das metas, das interpretações, das práticas, das explicações e do feedback que são parte integrante da aprendizagem (Gee, 2008, p.46).

Para uma melhor visualização e compreensão do conceito de DS e como ele inclui ambas as teorias, elaboramos a figura 1. 


\section{Domínio semiótico da vida cotidiana}

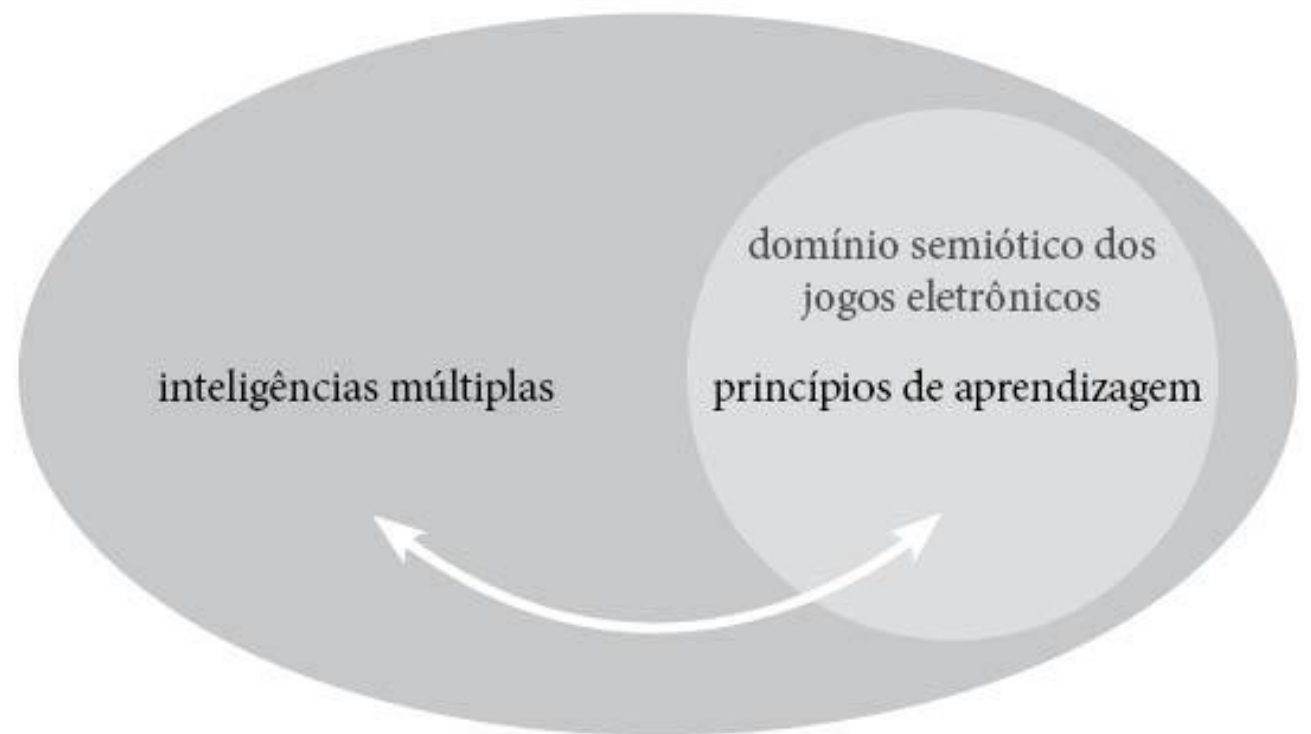

Figura 1 - Jogos eletrônicos, princípios de aprendizagem e inteligências múltiplas

\subsection{Os jogos eletrônicos e os princípios de aprendizagem}

A partir dos estudos de Gee (2003; 2005; 2008), percebe-se que os JEs podem, além de ensinar conteúdos, promover diversas outras importantes aprendizagens necessárias à vida contemporânea. De acordo com Gee (2008), como em qualquer experiência de aprendizagem, os JEs têm conteúdos que podem ser expressos por fatos, informações e habilidades que devem ser dominadas pelo jogador. No entanto, este conteúdo geralmente só é reconhecido como pertinente por não jogadores quando pertence a DS da vida cotidiana exterior aos JEs, como é caso da matemática e da história (Gee, 2008).

Segundo o autor, duas são as formas de ensinar algum conteúdo. A primeira é partir dele como foco principal e ensiná-lo diretamente, como geralmente ocorre na escola. A segunda é ensiná-lo indiretamente de forma subordinada a alguma outra coisa, como por meio dos bons JEs. Em acordo com as teorias recentes sobre a aprendizagem, Gee aponta a segunda opção como sendo a mais apropriada.

Gee (2003) aborda os JEs onde o jogador assume o papel de um personagem que se move em um mundo ficcional resolvendo diversos problemas e JEs onde o jogador constrói e mantém uma entidade complexa, como uma civilização ou exército. Estes são o que Gee denomina como "bons jogos", ou seja, títulos complexos de grande sucesso entre a crítica especializada e os jogadores que veiculam importantes PA, e por isso têm a capacidade de desenvolver diversas aprendizagens.

De acordo com o autor, se um JE veicula bons PA e é bem produzido, ele torna-se popular, obtendo boa recepção pela comunidade de jogadores. Dessa forma, os JEs têm que incluir e renovar seus PA e suas características interativas, mantendo-se desafiadores e divertidos. Para Gee, a teoria de aprendizagem existente nos bons JEs se adapta melhor ao mundo contemporâneo globalizado de alta tecnologia em que vivem as crianças e os 
adolescentes do que as teorias e as práticas de aprendizagem com as quais elas interagem na escola (Gee, 2003, p.7).

Gee (2003) descreve trinta e seis PA que podem estar veiculados aos JEs. A partir destes elaboramos o quadro comparativo da próxima seção. Destaque-se que nem todos os PA levantados pelo autor são necessariamente encontrados em um único JE; desta forma, há a possibilidade de que um JE veicule um ou mais destes princípios.

Na próxima seção, propomos, então, uma primeira aproximação de análise dos PA em relação às IMs, com o objetivo de levantar reflexões e questões sobre JEs, aprendizagem e educação.

\section{Construindo um modelo de aproximação das propostas}

O modelo aqui proposto apresenta os trinta e seis princípios de aprendizagem levantados por Gee (2003) e as oito IMs levantadas por Gardner (1983) em um quadro de modelo checklist. Neste, associa-se a proposta de Gee (2003) à de Gardner em uma matriz. Para tanto, partimos dos PA e de suas possíveis associações com as IMs. Vejamos a interação no Quadro 1 a seguir:

Quadro 1 - PA x IMs: aprendizagens possíveis

\begin{tabular}{|c|c|c|c|c|c|c|c|c|}
\hline \multirow{2}{*}{$\begin{array}{c}\text { Princípios de } \\
\text { Aprendizagem (PA) } \\
\text { - Gee (2003) }\end{array}$} & \multicolumn{8}{|c|}{ Inteligências Múltiplas (IMs) - Gardner $(1983,1993)$} \\
\hline & $\begin{array}{c}\text { A. } \\
\text { Linguística }\end{array}$ & $\begin{array}{c}\text { B. } \\
\text { Lógico- } \\
\text { Matemática }\end{array}$ & $\begin{array}{c}\text { C. } \\
\text { Espacial }\end{array}$ & $\begin{array}{c}\text { D. } \\
\text { Corporal- } \\
\text { Cinestésica }\end{array}$ & $\begin{array}{c}\text { E. } \\
\text { Musical }\end{array}$ & $\begin{array}{c}\mathrm{F} . \\
\text { Interpessoal }\end{array}$ & $\begin{array}{c}\text { G. } \\
\text { Intrapessoal }\end{array}$ & $\begin{array}{c}\mathrm{H} . \\
\text { Naturalista }\end{array}$ \\
\hline 1. Ativo e Crítico & $\mathrm{X}$ & $\mathrm{X}$ & $\mathrm{X}$ & $\mathrm{X}$ & $\mathrm{X}$ & $\mathrm{X}$ & $\mathrm{X}$ & $\mathrm{X}$ \\
\hline 2. Design & $\mathrm{X}$ & $\mathrm{X}$ & $\mathrm{X}$ & $\mathrm{X}$ & $\mathrm{X}$ & & & $\mathrm{X}$ \\
\hline 3. Semiótico & $\mathrm{X}$ & $\mathrm{X}$ & & $\mathrm{X}$ & $\mathrm{X}$ & & & $\mathrm{X}$ \\
\hline $\begin{array}{l}\text { 4.Domínios } \\
\text { Semióticos }\end{array}$ & $\mathrm{X}$ & $\mathrm{X}$ & $\mathrm{X}$ & $\mathrm{X}$ & $\mathrm{X}$ & $\mathrm{X}$ & $\mathrm{X}$ & $\mathrm{X}$ \\
\hline $\begin{array}{l}\text { 5.Pensamento em } \\
\text { Metanível sobre os } \\
\text { Domínios Semióticos }\end{array}$ & $\mathrm{X}$ & $\mathrm{X}$ & $\mathrm{X}$ & $\mathrm{X}$ & $\mathrm{X}$ & $\mathrm{X}$ & $\mathrm{X}$ & $\mathrm{X}$ \\
\hline $\begin{array}{ll}\text { 6.Suspensão } & \text { de } \\
\text { Consequências } & \\
\text { Psicossociais } & \end{array}$ & $\mathrm{X}$ & $\mathrm{X}$ & $\mathrm{X}$ & $\mathrm{X}$ & $\mathrm{X}$ & $\mathrm{X}$ & $\mathrm{X}$ & $\mathrm{X}$ \\
\hline $\begin{array}{l}\text { 7.Comprometimento } \\
\text { com a Aprendizagem }\end{array}$ & $\mathrm{X}$ & $\mathrm{X}$ & $\mathrm{X}$ & $\mathrm{X}$ & $\mathrm{X}$ & $\mathrm{X}$ & $\mathrm{X}$ & $\mathrm{X}$ \\
\hline 8. Identidade & $\mathrm{X}$ & $\mathrm{X}$ & $\mathrm{X}$ & $\mathrm{X}$ & $\mathrm{X}$ & $\mathrm{X}$ & $\mathrm{X}$ & $\mathrm{X}$ \\
\hline 9.Auto-Conhecimento & & & & & & & $\mathrm{X}$ & \\
\hline $\begin{array}{ll}\text { 10.Informação } & \text { de } \\
\text { Entrada Ampliada } & \\
\end{array}$ & $\mathrm{X}$ & $\mathrm{X}$ & $\mathrm{X}$ & $\mathrm{X}$ & $\mathrm{X}$ & $\mathrm{X}$ & $\mathrm{X}$ & $\mathrm{X}$ \\
\hline 11. Aquisição & $\mathrm{X}$ & $\mathrm{X}$ & $\mathrm{X}$ & $\mathrm{X}$ & $\mathrm{X}$ & $\mathrm{X}$ & $\mathrm{X}$ & $\mathrm{X}$ \\
\hline 12. Prática & $\mathrm{X}$ & $\mathrm{X}$ & $\mathrm{X}$ & $\mathrm{X}$ & $\mathrm{X}$ & $\mathrm{X}$ & $\mathrm{X}$ & $\mathrm{X}$ \\
\hline $\begin{array}{l}\text { 13.Aprendizagem } \\
\text { Contínua }\end{array}$ & $\mathrm{X}$ & $\mathrm{X}$ & $\mathrm{X}$ & $\mathrm{X}$ & $\mathrm{X}$ & $\mathrm{X}$ & $\mathrm{X}$ & $\mathrm{X}$ \\
\hline $\begin{array}{ll}\text { 14.Regime } & \text { de } \\
\text { Competência } & \end{array}$ & $\mathrm{X}$ & $\mathrm{X}$ & $\mathrm{X}$ & $\mathrm{X}$ & $\mathrm{X}$ & $\mathrm{X}$ & $\mathrm{X}$ & $\mathrm{X}$ \\
\hline 15. Sondagem & $\mathrm{X}$ & $\mathrm{X}$ & $\mathrm{X}$ & $\mathrm{X}$ & $\mathrm{X}$ & $\mathrm{X}$ & $\mathrm{X}$ & $\mathrm{X}$ \\
\hline 16.Rotas Múltiplas & $\mathrm{X}$ & $\mathrm{X}$ & $\mathrm{X}$ & $\mathrm{X}$ & $\mathrm{X}$ & $\mathrm{X}$ & $\mathrm{X}$ & $\mathrm{X}$ \\
\hline $\begin{array}{l}\text { 17.Conhecimento } \\
\text { Situado }\end{array}$ & $\mathrm{X}$ & $\mathrm{X}$ & $\mathrm{X}$ & $\mathrm{X}$ & $\mathrm{X}$ & $\mathrm{X}$ & $\mathrm{X}$ & $\mathrm{X}$ \\
\hline 18. Texto & $\mathrm{X}$ & & & & & & & \\
\hline 19. Intertextual & $\mathrm{X}$ & & & & & & & \\
\hline
\end{tabular}




\begin{tabular}{|c|c|c|c|c|c|c|c|c|}
\hline 20. Multimodal & $\mathrm{X}$ & $\mathrm{X}$ & $\mathrm{X}$ & $\mathrm{X}$ & $\mathrm{X}$ & & & $\mathrm{X}$ \\
\hline $\begin{array}{l}\text { 21.Inteligência } \\
\text { Material }\end{array}$ & $\mathrm{X}$ & $\mathrm{X}$ & $\mathrm{X}$ & $\mathrm{X}$ & $\mathrm{X}$ & & & $\mathrm{X}$ \\
\hline $\begin{array}{l}\text { 22. Conhecimento } \\
\text { Intuitivo }\end{array}$ & $\mathrm{X}$ & $\mathrm{X}$ & $\mathrm{X}$ & $\mathrm{X}$ & $\mathrm{X}$ & $\mathrm{X}$ & $\mathrm{X}$ & $\mathrm{X}$ \\
\hline 23. Subconjunto & $\mathrm{X}$ & $\mathrm{X}$ & $\mathrm{X}$ & $\mathrm{X}$ & $\mathrm{X}$ & $\mathrm{X}$ & $\mathrm{X}$ & $\mathrm{X}$ \\
\hline 24. Ampliação & $\mathrm{X}$ & $\mathrm{X}$ & $\mathrm{X}$ & $\mathrm{X}$ & $\mathrm{X}$ & $\mathrm{X}$ & $\mathrm{X}$ & $\mathrm{X}$ \\
\hline $\begin{array}{l}\text { 25.Amostra } \\
\text { Concentrada }\end{array}$ & $\mathrm{X}$ & $\mathrm{X}$ & $\mathrm{X}$ & $\mathrm{X}$ & $\mathrm{X}$ & $\mathrm{X}$ & $\mathrm{X}$ & $\mathrm{X}$ \\
\hline $\begin{array}{l}\text { 26.Habilidades Básicas } \\
\text { Iniciais }\end{array}$ & $\mathrm{X}$ & $\mathrm{X}$ & $\mathrm{X}$ & $\mathrm{X}$ & $\mathrm{X}$ & $\mathrm{X}$ & $\mathrm{X}$ & $\mathrm{X}$ \\
\hline $\begin{array}{l}\text { 27.Informação } \\
\text { Explícita “a pedido” e } \\
\text { “em tempo real” }\end{array}$ & $\mathrm{X}$ & $\mathrm{X}$ & $\mathrm{X}$ & $\mathrm{X}$ & $\mathrm{X}$ & $\mathrm{X}$ & $\mathrm{X}$ & $\mathrm{X}$ \\
\hline 28. Descoberta & $\mathrm{X}$ & $\mathrm{X}$ & $\mathrm{X}$ & $\mathrm{X}$ & $\mathrm{X}$ & $\mathrm{X}$ & $\mathrm{X}$ & $\mathrm{X}$ \\
\hline 29. Transferência & $\mathrm{X}$ & $\mathrm{X}$ & $\mathrm{X}$ & $\mathrm{X}$ & $\mathrm{X}$ & $\mathrm{X}$ & $\mathrm{X}$ & $\mathrm{X}$ \\
\hline $\begin{array}{l}\text { 30.Modelos Culturais } \\
\text { sobre o Mundo }\end{array}$ & $\mathrm{X}$ & $\mathrm{X}$ & $\mathrm{X}$ & $\mathrm{X}$ & $\mathrm{X}$ & $\mathrm{X}$ & $\mathrm{X}$ & $\mathrm{X}$ \\
\hline $\begin{array}{l}\text { 31.Modelos Culturais } \\
\text { sobre a Aprendizagem }\end{array}$ & $\mathrm{X}$ & $\mathrm{X}$ & $\mathrm{X}$ & $\mathrm{X}$ & $\mathrm{X}$ & $\mathrm{X}$ & $\mathrm{X}$ & $\mathrm{X}$ \\
\hline $\begin{array}{lr}\begin{array}{l}\text { 32.Modelos } \\
\text { sobre }\end{array} & \text { Culturais } \\
\text { Semióticos } & \end{array}$ & $\mathrm{X}$ & $\mathrm{X}$ & $\mathrm{X}$ & $\mathrm{X}$ & $\mathrm{X}$ & $\mathrm{X}$ & $\mathrm{X}$ & $\mathrm{X}$ \\
\hline 33. Distribuição & $\mathrm{X}$ & $\mathrm{X}$ & $\mathrm{X}$ & $\mathrm{X}$ & $\mathrm{X}$ & $\mathrm{X}$ & $\mathrm{X}$ & $\mathrm{X}$ \\
\hline 34. Dispersão & & & & & & $\mathrm{X}$ & & \\
\hline 35.Grupo de Afinidade & $\mathrm{X}$ & $\mathrm{X}$ & $\mathrm{X}$ & $\mathrm{X}$ & $\mathrm{X}$ & $\mathrm{X}$ & $\mathrm{X}$ & $\mathrm{X}$ \\
\hline 36.Participação Ativa & $\mathrm{X}$ & $\mathrm{X}$ & $\mathrm{X}$ & $\mathrm{X}$ & $\mathrm{X}$ & $\mathrm{X}$ & $\mathrm{X}$ & $\mathrm{X}$ \\
\hline
\end{tabular}

Como estratégia de explicação preliminar, apresentamos os resultados da análise do quadro por meio de seis grupos de itens, que provisoriamente denominamos de categorias e que listamos a seguir:

A) Categoria do grande grupo dos PA: esta é a maior das categorias por agrupar os PA de números $1,4,5,6,7,8,10,11,12,13,14,15,16,17,22,23,24,25,26,27,28,29,30$, 31, 32, 33, 34, 35 e 36. Estes PA foram agrupados aqui pois se relacionam com todas as IMs.

B) Categoria PA de design, multimodal e da inteligência material: reúne os PA 2, 20 e 21. Eles têm em comum o fato de não se associarem às inteligências intrapessoal e interpessoal.

C) Categoria PA semiótico: inclui apenas o PA 3, que não se associa às inteligências espacial, intrapessoal e interpessoal.

D) Categoria PA do auto-conhecimento: inclui apenas o PA 9, que se relaciona somente com a inteligência intrapessoal.

E) Categoria PA de texto e intertextual: reúne os PA 18 e 19, que se associam somente com a inteligência linguística.

F) Categoria PA da dispersão: inclui somente o PA 34, que se associa somente com a inteligência interpessoal.

Com base na análise do quadro, destacamos que os PA necessitam de muitas condições para serem desenvolvidos a partir do JE. Isto fica claro principalmente ao refletirmos sobre a maior das categorias (na lista em A, acima). Se estas condições forem atingidas, os PA talvez possam auxiliar no desenvolvimento das inteligências múltiplas. 
A análise aponta que, para desenvolver uma IM, o aprendiz precisa estar imerso em um ou mais DS que apresentem as condições e as qualidades necessárias para propiciar seu desenvolvimento. Por exemplo: não adianta o aprendiz de um esporte ter acesso a apenas uma modalidade esportiva para o pleno desenvolvimento de sua inteligência corporalcinestésica. É necessário que este tenha acesso e conhecimento de diversas modalidades esportivas, ou seja, diversos sub DS que fazem parte do DS esporte, além de existirem outros fatores extrínsecos e intrínsecos (motivação, lesões, materiais e treinamento adequados, etc.) para obter sucesso no domínio completo. Exemplos de vários atletas de destaque demonstram este fato, como pilotos de Fórmula 1, lutadores de MMA e atletas olímpicos. Como destaca Gee (2005) em relação à aprendizagem nos JEs, são princípios de aprendizagem que se encontram nos bons JEs. Estes princípios não necessariamente promovem a aprendizagem. Para que a aprendizagem no JE possa ocorrer e talvez desenvolver uma IM no DS da vida cotidiana, são necessários diversos fatores.

Neste sentido, o quadro revelou a existência de um binômio inexcedível: sem os PA, não há bons jogos, enquanto que, sem a valorização de um domínio no DS da vida cotidiana não há como avançar dentro daquele domínio. Assim, as IMs não podem se desenvolver plenamente em certos contextos culturais e os PA resultam sem valor nesses contextos. Muito esclarecedor, para essa questão, é o que vemos na categoria que denominamos de grande grupo dos PA (em A, acima): este grupo reúne vinte e nove dos trinta e seis PA.

Além disso, a matriz revelou a importância da inteligência interpessoal na aprendizagem ao mostrar que esta se associa a trinta dos trinta e seis PA (em A e F, acima). A inteligência interpessoal é desenvolvida por meio do trabalho cooperativo, do envolvimento comunitário, de simulações de grandes grupos, de dedicação a questões sociais, etc. Justamente a importância da inteligência interpessoal, conforme observa Gardner (1983:352), vem sendo reduzida na cena educacional contemporânea: a sensibilidade aos outros indivíduos como indivíduos, a capacidade de colaborar com os outros assume cada vez menos importância. Desse modo, acreditamos que os resultados da comparação entre as teorias colocam em questão as formas como concebemos e gerenciamos a educação em seus diversos âmbitos. Por essa razão, acreditamos que futuras análises do cruzamento das teorias estudadas aqui poderão nos auxiliar tanto na utilização dos JEs como proposta pedagógica, como na reflexão sobre a educação.

\section{Considerações finais}

A associação entre ambas as teorias pareceu-nos produtiva para refletir sobre os JEs e a aprendizagem em geral. Em primeiro lugar, devemos observar que nem todos os JEs promovem a totalidade dos PA. Isto acontece porque há muitos fatores no DS da vida cotidiana que podem dificultar a aprendizagem e o desenvolvimento das IMs. E vemos isto ocorrer até mesmo quando o JE veicula os PA ou as condições básicas para desenvolvê-los, o que demonstra a íntima associação entre os princípios e as inteligências.

Em segundo lugar, a leitura do quadro revelou que a inteligência interpessoal está associada a trinta PA. O que demonstra a complexidade inerente ao aprendizado em geral e consequentemente os desafios que a educação contemporânea deve enfrentar. De fato, o 
estudo da interação entre as teorias, como proposto no quadro, pode nos auxiliar a pensar novas formas de ensino e aprendizagem em diversos âmbitos, destacando-se o lazer.

Parece-nos que a importância da teoria de Gee está em destacar a relevância dos JEs culturalmente e para a aprendizagem, enquanto a teoria da aprendizagem de Gardner ressalta a relação entre desenvolvimento de habilidades e existência de condições propícias (ambiente, mentores, valorização cultural, etc.). As habilidades/inteligências são valorizadas de modo diferente entre as diversas culturas.

Finalmente, acreditamos que as particularidades encontradas no quadro proposto podem servir ainda como subsídio para o desenvolvimento de JEs, por exemplo, ao apontar quais PA são associados a todas as IMs e quais se associam unicamente a certas inteligências. Os JEs representam, a partir da visão proposta por Gee, oportunidades de aprendizagem direta e indireta de conteúdos e de habilidades no DS da vida cotidiana, tendo em vista sua ligação intrínseca com a maioria das IMs. Seja como for, reconhecemos que o quadro pode ser interpretado a partir de diferentes pontos de vista e seus objetivos. Enfim, observamos que futuras divulgações dos resultados desta pesquisa poderão tratar de outros aspectos encontrados no quadro.

\footnotetext{
${ }^{1}$ Informações sobre este jogo em: http://en.wikipedia.org/wiki/OXO. Acessado dia 30/10/2012. Vídeo de OXO disponível no site: http://www.youtube.com/watch?v=vCTRWD3DFsA. Acessado dia 30/10/2012.

${ }^{2}$ A classificação em gêneros interativos de JEs utilizada neste texto foi proposta por Wolf (2005).
} 


\section{Referências bibliográficas}

ARMSTRONG, T. Multiple intelligences in the classroom. Alexandria (VA): ASCD, 2009.

GARDNER, H. Frames of mind. The theory of multiple intelligences. Nova Iorque: Basic Books, 1983.

GARDNER, H. Multiple intelligences: new horizons. Nova Iorque: Basic Books, 2006. (1. ed. 1993)

GEE, J. P. What video games have to teach us about learning and literacy. Nova Iorque: Palgrave MacMillan, 2003.

GEE, J. P. Good videos games and good learning. Phi Kappa Phi Forum, v. 85, n. 2, 2005.

GEE, J. P. Video Games, learning, and “content”. In: MILLER, C. T. (Org.). Games: purpose and potential in education. Nova York: Springer, 2008

JUUL, J. Half-real: video games between real rules and fictional worlds. Cambridge (MA): MIT Press, 2005.

SHAFFER, D. W. How computer games help children learn. Nova Iorque: Palgrave, 2006.

WOLF, M. J. P. The medium of the video game. Austin: University of Texas Press, 2001. WREN, C.; WREN, T. The capacity to learn. In: CURREN, R. (Ed.) A companion to the philosophy of education. Londres: Blackwell, 2003, p. 246-259. 